\title{
Video Article \\ Surface Functionalization of Metal-Organic Frameworks for Improved Moisture Resistance
}

\author{
Javier Castells-Gil ${ }^{1}$, Fernando Novio ${ }^{2}$, Natalia M. Padial ${ }^{1}$, Sergio Tatay ${ }^{1}$, Daniel Ruíz-Molina ${ }^{2}$, Carlos Martí-Gastaldo ${ }^{1}$ \\ ${ }^{1}$ Instituto de Ciencia Molecular (ICMol), Universitat de València \\ ${ }^{2}$ Catalan Institute of Nanoscience and Nanotechnology (ICN2), CSIC and The Barcelona Institute of Science and Technology
}

Correspondence to: Carlos Martí-Gastaldo at carlos.marti@uv.es

URL: https://www.jove.com/video/58052

DOI: doi: $10.3791 / 58052$

Keywords: Chemistry, Issue 139, Metal-organic frameworks, surface functionalization, water stability, catecholase biomimetics, hydrophobic coating, functionalized catechols

Date Published: 9/5/2018

Citation: Castells-Gil, J., Novio, F., Padial, N.M., Tatay, S., Ruíz-Molina, D., Martí-Gastaldo, C. Surface Functionalization of Metal-Organic Frameworks for Improved Moisture Resistance. J. Vis. Exp. (139), e58052, doi:10.3791/58052 (2018).

\section{Abstract}

\begin{abstract}
Metal-organic frameworks (MOFs) are a class of porous inorganic materials with promising properties in gas storage and separation, catalysis and sensing. However, the main issue limiting their applicability is their poor stability in humid conditions. The common methods to overcome this problem involve the formation of strong metal-linker bonds by using highly charged metals, which is limited to a number of structures, the introduction of alkylic groups to the framework by post-synthetic modification (PSM) or chemical vapour deposition (CVD) to enhance overall hydrophobicity of the framework. These last two usually provoke a drastic reduction of the porosity of the material. These strategies do not permit to exploit the properties of the MOF already available and it is imperative to find new methods to enhance the stability of MOFs in water while keeping their properties intact. Herein, we report a novel method to enhance the water stability of MOF crystals featuring $\mathrm{Cu}_{2}\left(\mathrm{O}_{2} \mathrm{C}\right)_{4} \mathrm{paddle}$ wheel units, such as HKUST (where HKUST stands for Hong Kong University of Science \& Technology), with the catechols functionalized with alkyl and fluoro-alkyl chains. By taking advantage of the unsaturated metal sites and the catalytic catecholase-like activity of $\mathrm{Cu}$ "ions, we are able to create robust hydrophobic coatings through the oxidation and subsequent polymerization of the catechol units on the surface of the crystals under anaerobic and water-free conditions without disrupting the underlying structure of the framework. This approach not only affords the material with improved water stability but also provides control over the function of the protective coating, which enables the development of functional coatings for the adsorption and separations of volatile organic compounds. We are confident that this approach could also be extended to other unstable MOFs featuring open metal sites.
\end{abstract}

\section{Video Link}

The video component of this article can be found at https://www.jove.com/video/58052/

\section{Introduction}

Metal-organic frameworks are a class of crystalline porous materials built from inorganic metallic components, typically named secondary building units (SBUs), held together by polytopic organic ligands through coordinative bonds. The self-assembly of the these SBUs with the organic linkers enables the formation of extended 3D porous structures with very high surface areas and promising applications in the fields of gas storage and separation ${ }^{1,2}$, catalysis and sensing ${ }^{3}$. However, the main limitation for their applicability is their poor stability in water ${ }^{4,5}$ as most of them incorporate divalent metals in their structure that results in labile coordination bonds, as those encountered in classical materials like MOF-56 $5^{6}$ HKUST ${ }^{7}$.

Common approaches to solve this problem involve on the one hand, the creation of stronger coordination bonds by the use of highly charged metals, such as $\mathrm{Zr}$ or Ti(IV), basic $\mathrm{N}$-donor ligands ${ }^{7,8}$ or ligands incorporating acids and basic sites ${ }^{9}$. However, this method is limited to new materials and does not allow to enhance the stability of MOFs already available. On the other hand, the approaches to improve the stability of the already known materials use the post-synthetic modification methods to introduce hydrophobic moieties in the empty space by post-synthetic modification of the linker ${ }^{10,11}$ or by chemical vapour deposition (CVD) ${ }^{12}$. Unfortunately, the stability of these methods comes at the expenses of a drastic reduction in the porosity of the material and the use of sophisticated instrumentation. The recent use of modified phosphonic acids, such as 1,2-dioleoyl-sn-glycero-3-phosphate (DOPA) ${ }^{13}$ or $n$-octadecylphosphonic acid (OPA) ${ }^{14}$, to impart hydrophobicity in known $\mathrm{Zr}(\mathrm{IV}) \mathrm{MOF}$ should also be highlighted.

Catechol compounds, such as dopamine, have been extensively used to functionalize a broad range of materials through the formation of polydopamine ${ }^{15}$. However, the formation of these coatings is limited to the use of aqueous buffered solutions for slightly basic solutions which are not suitable for MOFs with labile bonds. Bortoluzzi et al. recently reported that polydopamine can be produced in solution by a binuclear $\mathrm{Cu}(\mathrm{II})$ complex featuring $\mathrm{Cu}_{2}(\mu-\mathrm{O})$ as a catalytic ${ }^{16}$ centre which displays catecholase-like catalytic activity reminiscent of natural enzymes such as catechol oxidase ${ }^{17}$ and tyrosinase ${ }^{18}$. More recently, we have shown how a MOF based on Cu(II) paddle-wheel SBUs connected through trimesate linkers, known as HKUST, can be protected from hydrolytic degradation by the polymerization of functionalized catechols, such as 4hepatdecyl-catechol (hdcat) or fluorinated-4-undecylcatechol (fdcat), on the surface of the crystals ${ }^{19}$. This simple method proves how efficient 
functional coatings can be synthesized under mild conditions regardless of the functionality of the catechol and without the use of buffer solutions that could compromise the stability of the framework, due to the biomimetic catalytic activity of the $\mathrm{Cu}(\mathrm{II})$ units. We believe that this new method could enable the formation of functional coatings that, besides protecting from hydrolytic degradation, might enable selective adsorption of chiral molecules or volatile organic compounds.

\section{Synthetic Procedure of hdcat@HKUST}

NOTE: The entire process must be performed inside a glove-box in order to avoid any contact with the ambient moisture. Accordingly, all the reagents and solvents used must be dry and stored in the glove-box.

1. Bring an open $4 \mathrm{~mL}$ glass vial, two spatulas and a $1 \mathrm{~mL}$ micropipette into the glove-box.

2. Transfer $50 \mathrm{mg}$ of hdcat into the glass vial. NOTE: In some cases, an anti-static gun may be necessary in order to avoid the undesirable effects of static electricity.

3. Place $1 \mathrm{~mL}$ of anhydrous chloroform in the glass vial containing hdcat. NOTE: Not all hdcat may be dissolved entirely at room temperature, but it dissolves very rapidly when the vial is placed in the oven in the next steps.

4. Place $10 \mathrm{mg}$ of HKUST in the chloroform solution containing hdcat and seal the vial tightly.

5. Take the vial out of the glove-box and sonicate the suspension of HKUST and hdcat in chloroform for a few seconds to homogenize the solution.

NOTE: Do not expose the contents of the vial to ambient air as the introduction of $\mathrm{O}_{2}$ in the reaction media could drive the polymerization of the catechol units in solution rather than on the surface of the crystals ${ }^{15}$.

6. Place the vial in the oven at $70^{\circ} \mathrm{C}$ overnight. Make sure that the vial is tightly sealed in order to avoid the evaporation of the chloroform during the reaction (boiling point $\left(\mathrm{CHCl}_{3}\right)=61.2^{\circ} \mathrm{C}$ ).

NOTE: In some cases, a Teflon strip surrounding the screw cap may be helpful. This protocol requires a preheated oven at $70{ }^{\circ} \mathrm{C}$. The temperature should not be higher than $70^{\circ} \mathrm{C}$, as amorphous products could be obtained otherwise.

\section{Washing Procedure of hdcat@HKUST}

1. Take the vial out of the oven after being overnight at $70^{\circ} \mathrm{C}$ and transfer it to the glove-box along with a $15 \mathrm{~mL}$ centrifuge tube.

2. Transfer the contents of the vial to the centrifuge tube inside the glove-box using fresh anhydrous chloroform.

3. Separate the coated material hdcat@HKUST by centrifugation ( 3354 x g, 1 min). Make sure that the centrifuge tube is tightly capped as it must be taken out of the glove-box in order to centrifuge the material.

4. Introduce the centrifuge tube rapidly in the glove-box after the centrifugation.

5. Extract the supernatant carefully using a dropper and store it in a clean $40 \mathrm{~mL}$ glass vial.

6. Suspend the coated material in $3 \mathrm{~mL}$ of anhydrous $\mathrm{CHCl}_{3}$ in order to remove possible polymerized catechol units that are not attached to the surface of the crystals.

7. Repeat steps 2.3-2.6 three times

8. Suspend the coated material in $3 \mathrm{~mL}$ of anhydrous methanol.

9. Repeat steps 2.3-2.6 three times but using anhydrous methanol in order to remove unreacted hdcat molecules. NOTE: Do not throw away the hdcat solutions as the product can be recovered by slow evaporation of the solutions in the glove-box and reused.

10. Transfer the washed hdcat@HKUST to a glass vial using anhydrous methanol and wait until the coated solid settles at the bottom of the vial.

11. Take out the supernatant and let the powder dry at room temperature in the glove-box.

\section{Synthetic Procedure of fdcat@HKUST}

NOTE: The entire process must be performed inside a glove-box in order to avoid any contact with the ambient moisture. Accordingly, all the reagents and solvents used must be dry and stored in the glove-box.

1. Introduce an open $4 \mathrm{~mL}$ glass vial, two spatulas and a $1 \mathrm{~mL}$ micropipette into the glove-box.

2. Place $50 \mathrm{mg}$ of fdcat inside the glass vial.

NOTE: In some cases, an anti-static gun may be necessary in order to avoid the undesirable effects of the static electricity.

3. Place $1 \mathrm{~mL}$ of anhydrous chloroform in the glass vial containing fdcat.

NOTE: Not all the fdcat may be dissolved entirely at room temperature, but it dissolves very rapidly when the vial is placed in the oven in the next steps.

4. Place $10 \mathrm{mg}$ of HKUST in the chloroform solution containing fdcat and seal the vial tightly.

5. Take the vial out of the glove-box and sonicate the suspension of HKUST and fdcat in chloroform for a few seconds to homogenize the solution.

NOTE: Do not expose the contents of the vial to ambient air in any case as the introduction of $\mathrm{O}_{2}$ in the reaction media could drive the polymerization of the catechol units in solution rather than on the surface of the crystals ${ }^{15}$.

6. Place the vial in the oven at $70^{\circ} \mathrm{C}$ overnight. Make sure that the vial is tightly sealed in order to avoid the evaporation of chloroform during the reaction (boiling point $\left(\mathrm{CHCl}_{3}\right)=61.2^{\circ} \mathrm{C}$ ).

NOTE: In some cases, a teflon strip surrounding the screw cap may be helpful. This protocol requires a preheated oven at $70{ }^{\circ} \mathrm{C}$. The temperature should not be higher than $70^{\circ} \mathrm{C}$, as amorphous products could be obtained otherwise. 


\section{Washing Procedure of fdcat@HKUST}

1. Take the vial out of the oven after being overnight at $70^{\circ} \mathrm{C}$ and transfer it to the glove-box along with a $15 \mathrm{~mL}$ centrifuge tube.

2. Transfer the contents of the vial to the centrifuge tube inside the glove-box using fresh anhydrous chloroform.

3. Separate the coated material fdcat@HKUST by centrifugation ( 3354 x g, 1 min). Make sure that the centrifuge tube is tightly capped as it must be taken out of the glove-box in order to centrifuge the material.

4. Introduce the centrifuge tube rapidly into the glove-box after the centrifugation.

5. Extract the supernatant carefully using a dropper and store it in a clean $40 \mathrm{~mL}$ glass vial.

6. Suspend the coated material in $3 \mathrm{~mL}$ of anhydrous $\mathrm{CHCl}_{3}$ in order to remove possible polymerized catechol units that are not attached to the surface of the crystals.

7. Repeat steps 4.3-4.6 three times.

8. Suspend the coated material in $3 \mathrm{~mL}$ of anhydrous methanol.

9. Repeat steps 4.3-4.6 three times but using anhydrous methanol in order to remove unreacted fdcat molecules. NOTE: Do not throw away the fdcat solutions as the product can be recovered by slow evaporation of the solutions in the glove-box and reused.

10. Transfer the washed fdcat@HKUST to a glass vial using anhydrous methanol and wait until the coated solid settles at the bottom of the vial.

11. Take out the supernatant and let the powder dry at room temperature in the glove-box.

\section{Representative Results}

All the reagents and materials were stored in the glove-box and used as received without any further purification unless otherwise stated. The entire process is carried out in a glove-box in order to avoid contact with humidity that could degrade the uncoated material.

In order to ensure the reproducibility during the experiments, commercially available HKUST with an average particle size close to 40-50 $\mu \mathrm{m}$ (Figure 1), was used as previous studies suggested that the particle is important for reproducible surface density functionalization ${ }^{20}$.

The crystals of HKUST are suspended in anhydrous chloroform solutions of hdcat or fdcat (Figure 1c) in the glove-box. The glass vials were tightly capped and taken out of the glove-box and sonicated for a few seconds to homogenize the suspension. Then the mixtures were heated at $70{ }^{\circ} \mathrm{C}$ overnight in a preheated oven under static conditions. The solids were separated by centrifugation and rinsed with chloroform ( $\left.\mathrm{x} 3\right)$ and methanol $(x 3)$ in order to remove unattached polymerized units and unreacted catechol molecules, respectively ${ }^{15}$.

The first demonstration of the surface modification of the crystals is their increased hydrophobicity when they are soaked in water (Figure 2). In comparison with the bare HKUST, which immediately sinks to the bottom of the vial, hdcat@HKUST and fdcat@HKUST can stand on water for several days without sinking. Contact angle (CA) measurements indeed confirm the superior hydrophobicity of hdcat@HKUST and fdcat@HKUST with CA values of $107 \pm 1^{\circ}$ and $124 \pm 1^{\circ}$, respectively, compared to HKUST which was highly hydrophilic (Figure 2).

Comparison of the FT-IR spectra of HKUST before and after the coating process to those of hdcat and fdcat suggested the correct incorporation of the catecholate molecules onto the crystal. In the case of hdcat@HKUST (Figure 3a), the bands corresponding to alkane C-H stretching vibrations $\left(3000-2800 \mathrm{~cm}^{-1}\right)$ of the alkylic chain of hdcat can be observed, which are not present in the bare HKUST. For fdcat@HKUST (Figure 3b), the new bands appearing are those of the alkane C-F stretching vibrations $\left(1250-1100 \mathrm{~cm}^{-1}\right)$ which are not observed in HKUST. As estimated from thermogravimetric analysis in our previous work ${ }^{19}$, the catecholate grafting represented a $3.1 \%$ and $2.6 \%$ for hdcat@HKUST and fdcat@HKUST, respectively.

Scanning-electron microscopy (SEM) images of hdcat@HKUST and fdcat@HKUST shows an external corrugated layer of ca.600 nm surrounding the crystals. These results suggested an effective polymerization of the hdcat and fdcat molecules on the surface of the crystals while respecting their morphology (Figure 4a). This was further confirmed by X-ray photoelectron spectroscopy (XPS) measurements which showed the presence of $\mathrm{Cu}(\mathrm{I})$ and $\mathrm{Cu}(\mathrm{II})$ at 933 and $935 \mathrm{eV}$, respectively, in hdcat@HKUST and fdcat@HKUST, which we attributed to the reaction of the catechol moieties by $\mathrm{Cu}_{2}$ units on the surface and subsequent polymerization (Figure $4 \mathbf{b}$ ). As detailed in our previous study, NMR spectra of the digested hdcat@HKUST and fdcat@HKUST also confirms that the material surrounding the crystals are indeed the polymerized catechol molecules ${ }^{15,19}$

The formation of catecholate coatings on HKUST was found to proceed with no impact over the crystalline structure of HKUST as confirmed by powder X-ray diffraction measurements (PXRD, Figure 4c). This was also confirmed by porosity measurements at $77 \mathrm{~K}$ using $\mathrm{N}_{2}$ as adsorbent (Figure 4d), which showed that hdcat@HKUST and fdcat@HKUST retain their surface area with minor variations after the coating process. This result also suggests that the polymerization reaction occurs only on the surface of the crystals rather than in the pores of the material. 

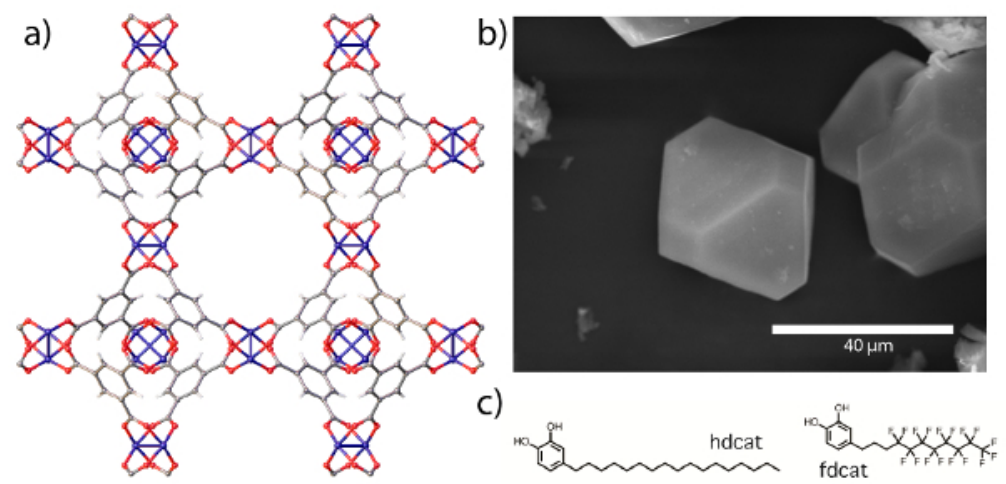

Figure 1: Schematic representation of the materials. (a) Crystalline structure of HKUST, (b) SEM micrograph of a HKUST crystal and (c) chemical structure of the functionalized catechols. Please click here to view a larger version of this figure.

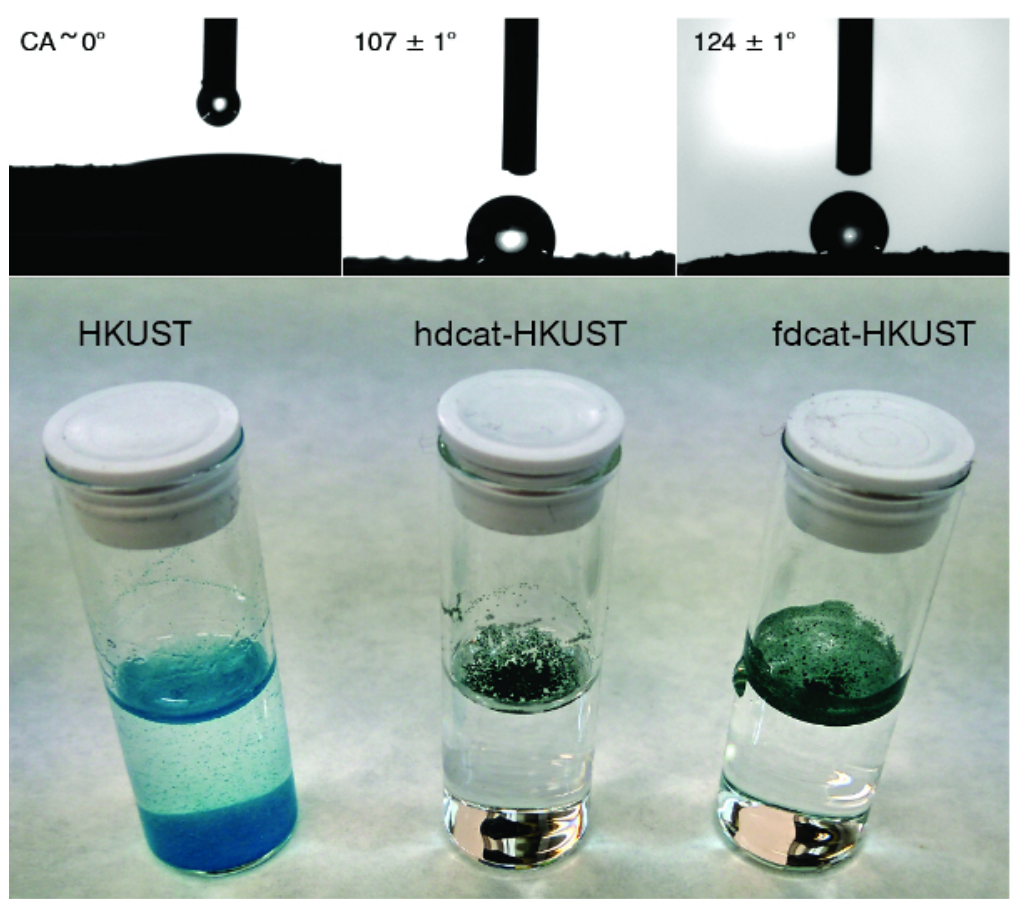

Figure 2: Hydrophobicity of the coated materials. Contact angle values of the bare HKUST, hdcat@HKUST and fdcat@HKUST and picture showing the difference in hydrophobicity of the modified solids compared to HKUST. This figure has been adapted with permission from ref. 19. Please click here to view a larger version of this figure.
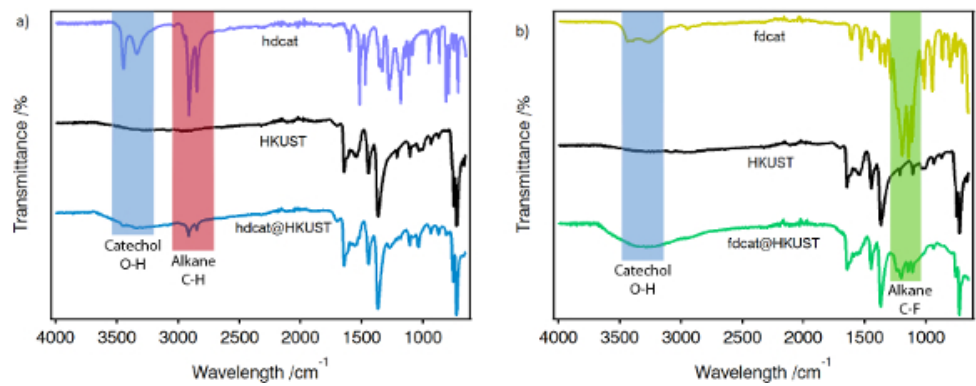

Figure 3: Chemical characterization of hdcat@HKUST and fdcat@HKUST. Fourier-transformed infrared (FT-IR) spectra of hdcat@HKUST with HKUST and hdcat (a), and fdcat@HKUST with HKUST and fdcat (b). This figure has been adapted with permission from ref. 19. Please click here to view a larger version of this figure. 

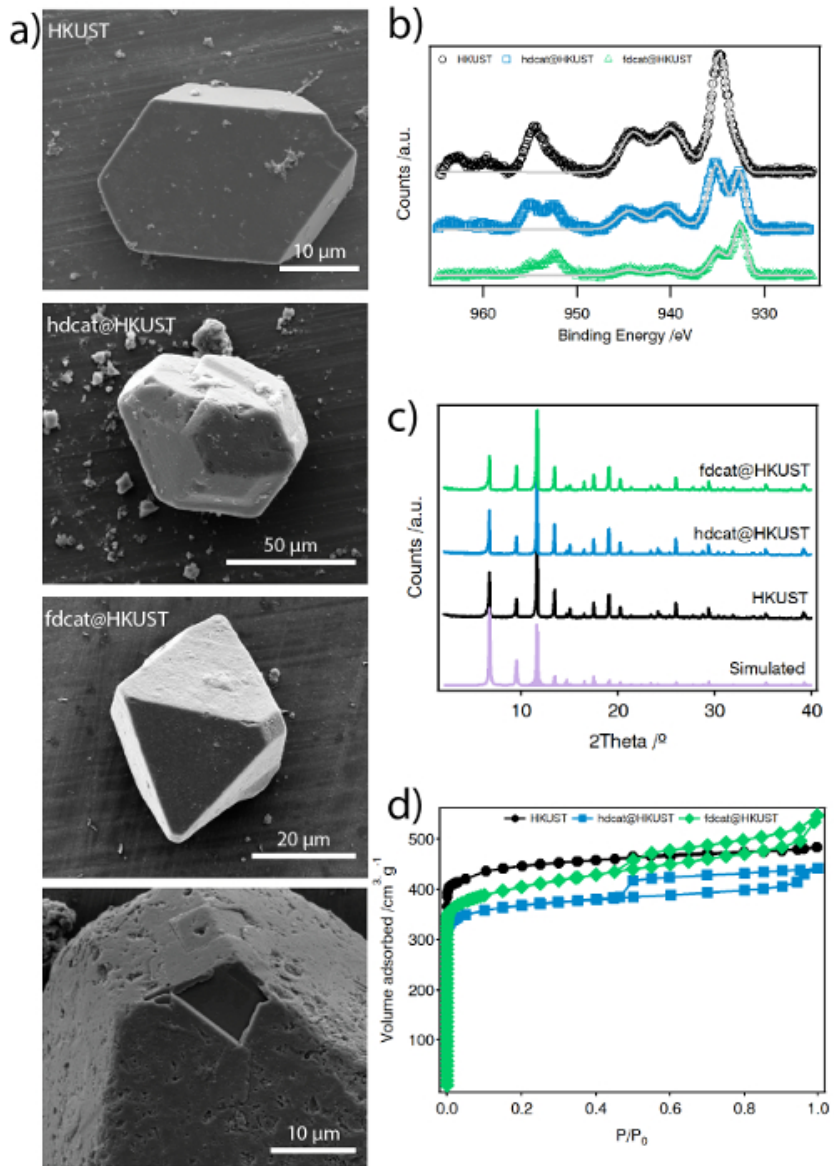

Figure 4: Effect of the catecholate coating on the properties of HKUST. (a) SEM images of HKUST, hdcat@HKUST and fdcat@HKUST crystals. (b) Cu 2p high-resolution XPS spectra, (c) PXRD patterns compared to the simulated PXRD of HKUST and (d) $\mathrm{N}_{2}$ isotherms at $77 \mathrm{~K}$ of the solids before and after the coating process. This figure has been adapted with permission from ref. 19. Please click here to view a larger version of this figure.

\section{Discussion}

The method reported in this work provides a simple and effective approach for the surface modification of MOF crystals by direct reaction with synthetic catechols under mild conditions regardless the functionality of the chain. Unlike the conventional approach of producing polydopaminelike coatings, this route can be performed in anhydrous and anaerobic conditions and without any base addition that could compromise the stability of the MOF. Methanol and chloroform were first chosen based on previous works ${ }^{14,20}$ and due to the high solubility of the catechol molecules in these solvents. However, methanol was rapidly discarded due to the low catechol loadings obtained in HKUST (ca 1.2 wt \% for hdcat), as compared to those obtained with chloroform (ca. $3.6 \%$ for hdcat), based on previous thermogravimetric analyses ${ }^{19}$. Therefore, the solvent does not play an innocent role as different solvents can yield different catechol loadings. It is important to highlight that the process must be carried out in an oxygen-free atmosphere as oxygen can promote the oxidative polymerization of the catechol molecules in solution and not on the surface of the material. The surface modification of HKUST with hdcat or fdcat can be directly observed by contact angle measurements (Figure 2), which showed the change from hydrophilic to highly hydrophobic in both hdcat@HKUST and fdcat@HKUST, and infrared spectroscopy (Figure 3) which showed the characteristic vibrational bands of the catechol moieties in the modified solids.

The functionalization of the framework occurs without any appreciable loss of crystallinity nor sorption properties of the material (Figures $4 \mathbf{c}$-d). Further inspection of hdcat@HKUST and fdcat@HKUST crystals by scanning-electron microscopy reveals a rougher surface compared to bare HKUST. A treatment of the modified crystals in chloroform under thorough sonication allowed part of the polycatecholate coating to be peeled off (Figure 4a) revealing part of the original crystal, which also served to determine an approximate thickness of the coating layer (ca. $600 \mathrm{~nm})^{19}$. The formation of this polycatechol coatings is attributed to the biomimetic catalytic activity of the $\mathrm{Cu}(\mathrm{II})$ species present on the surface of the HKUST crystals on the oxidation of catechol molecules, similar to the enzymatic activity of catechol oxidase ${ }^{17}$, as it also confirmed by the XPS measurements which show the presence of $\mathrm{Cu}(\mathrm{I})$ on the surface of the crystals as a result of the oxidative polymerization process. Unlike other works describing the surface functionalization of MOFs crystals with polymeric matrices ${ }^{12}$, which make use of sophisticated instrumentation, this methodology takes advantage of the MOF features, such as the open metal sites present in HKUST, in order to trigger the polymerization of the catechol molecules under mild conditions.

This approach not only helps to improve the moisture tolerance of the material ${ }^{19}$, but also affords control over the functionality of the grafting around the crystals, as it can be manipulated by convenient choice of the functionalized catechol. We believe that this method will provide an interesting approach not only for known Cu-MOF materials, but also for other MOFs featuring open metal sites, which would be able to 
incorporate novel functionalities that were not present in the non-functionalized solid, such as the adsorption of chiral molecules or volatile organic compounds. This can be achieved by suitable choice of the functionality present in the catechol molecule.

\section{Disclosures}

The authors have nothing to disclose.

\section{Acknowledgements}

This work was supported by the EU (ERC Stg Chem-fs-MOF 445 714122), Spanish MINECO (Unit of Excellence MDM-2015-0538), and the Generalitat Valenciana 447 (Grant GV/2016/137). C.M.-G. and J.C.-G. thank the Spanish 448 MINECO for a Ramón y Cajal Fellowship and FPI Scholarship 449 (CTQ2014-59209-P), respectively. N.M.P. thanks the Junta de 450 Andalucía for a postdoctoral fellowship P10-FQM-6050. F.N. and 451 D.R.M. are also grateful to the financial support offered by 452 Project MAT2015-70615-R from the Spanish Government and 453 by FEDER funds. The ICN2 is funded by the CERCA programme/Generalitat de Catalunya and supported by the Severo Ochoa programme of the Spanish Ministry of Economy, Industry and Competitiveness (MINECO, grant no. SEV-2013-0295).

\section{References}

1. Banerjee, D. et al. Metal-organic framework with optimally selective xenon adsorption and separation. Nature Communications. 7, (2016).

2. Elsaidi, S.K. et al. Hydrophobic pillared square grids for selective removal of $\mathrm{CO}_{2}$ from simulated flue gas. Chemical Communications. 51 (85), 15530-15533, (2015).

3. Furukawa, H., Cordova, K.E., O'Keeffe, M., Yaghi, O.M. The chemistry and applications of metal-organic frameworks. Science (New York, N.Y.). 341 (6149), 1230444, (2013).

4. Howarth, A.J. et al. Chemical, thermal and mechanical stabilities of metal-organic frameworks. Nature Reviews Materials. 1 (3), 15018, (2016).

5. Burtch, N.C., Jasuja, H., Walton, K.S. Water Stability and Adsorption in Metal-Organic Frameworks. Chem Rev. 114 (20), 10575-10612, (2014).

6. Guo, P., Dutta, D., Wong-Foy, A.G., Gidley, D.W., Matzger, A.J. Water Sensitivity in Zn4O-Based MOFs is Structure and History Dependent. Journal of the American Chemical Society. 137 (7), 2651-2657, (2015).

7. Gao, W.Y. et al. Remote stabilization of copper paddlewheel based molecular building blocks in metal-organic frameworks. Chemistry of Materials. 27 (6), 2144-2151, (2015).

8. Devic, T., Serre, C. High valence 3p and transition metal based MOFs. Chemical Society Reviews. 43 (43), 6097-6115, (2014).

9. He, H. et al. A Stable Metal-Organic Framework Featuring a Local Buffer Environment for Carbon Dioxide Fixation. Angewandte Chemie International Edition. 57 (17), 4657-4662, (2018).

10. Nguyen, J.G., Cohen, S.M. Moisture-resistant and superhydrophobic metal-organic frameworks obtained via postsynthetic modification. Journal of the American Chemical Society. 132 (13), 4560-4561 (2010).

11. Sun, Q. et al. Imparting amphiphobicity on single-crystalline porous materials. Nature Communications. 7, 13300, (2016).

12. Decoste, J.B., Peterson, G.W., Smith, M.W., Stone, C.A., Willis, C.R. Enhanced stability of Cu-BTC MOF via perfluorohexane plasmaenhanced chemical vapor deposition. Journal of the American Chemical Society. 134 (3), 1486-1489, (2012).

13. Wang, S. et al. Surface-specific functionalization of nanoscale metal-organic frameworks. Angewandte Chemie - International Edition. 54 (49), 14738-14742, (2015).

14. Sun, Y. et al. A molecular-level superhydrophobic external surface to improve the stability of metal-organic frameworks. Journal of Materials Chemistry A. 5 (35), 18770-18776, (2017).

15. Saiz-Poseu, J. et al. Versatile Nanostructured Materials via Direct Reaction of Functionalized Catechols. Advanced Materials. 25 (14), 2066-2070, (2013).

16. de Oliveira, J.A.F. et al. Dopamine polymerization promoted by a catecholase biomimetic $\mathrm{Cu} \mathrm{II}(\mu-\mathrm{OH}) \mathrm{Cu}$ Ilcomplex containing a triazinebased ligand. Dalton Transactions. 45 (39), 15294-15297, (2016).

17. Koval, I.A., Gamez, P., Belle, C., Selmeczi, K., Reedijk, J. Synthetic models of the active site of catechol oxidase: mechanistic studies. Chemical Society Reviews. 35 (9), 814, (2006).

18. Yang, J., Cohen Stuart, M.A., Kamperman, M. Jack of all trades: versatile catechol crosslinking mechanisms. Chemical Society Reviews 43 (43), 8271-8298, (2014).

19. Castells-Gil, J., Novio, F., Padial, N.M., Tatay, S., Ruíz-Molina, D., Martí-Gastaldo, C. Surface Functionalization of Metal-Organic Framework Crystals with Catechol Coatings for Enhanced Moisture Tolerance. ACS Applied Materials and Interfaces. 9 (51), 44641-44648, (2017).

20. Wang, S. et al. Surface-Specific Functionalization of Nanoscale Metal-Organic Frameworks. Angewandte Chemie. 127 (49), 14951-14955, (2015). 